\title{
Nonstationary Nonplanar Free Motions of an Orbiting String with Multiple Internal Resonances
}

\author{
ANGELO DI EGIDIO $^{1}$, ANGELO LUONGO $^{1}$ and FABRIZIO VESTRONI ${ }^{2}$ \\ ${ }^{1}$ Università di L'Aquila, Dipartimento di Ingegneria delle Strutture, delle Acque e del Terreno; 67040 Monteluco \\ Roio, L'Aquila, Italy \\ ${ }^{2}$ Università di Roma 'La Sapienza', Dipartimento di Ingegneria Strutturale e Geotecnica, Via Eudossiania 18; \\ 00184 Roma, Italy
}

\begin{abstract}
The paper discusses the nonlinear free dynamics of an orbiting string satellite system. The focus is on the transversal oscillations, which are governed by two partial integro-differential equations in two transversal displacement components with quadratic nonlinearities. The system is weakly nonlinear but in practice works in conditions of simultaneous internal resonance. The investigation focuses on nonstationary motions arising from perturbed steady-state nonplanar oscillations. A four-mode model is used to study the problem: two modes are necessary to describe the basic oscillation and at least two other modes are involved in the resonance phenomena when the motion is perturbed. The multiple time scales method is used to obtain the equations that govern the amplitude and phase modulations. For increasing levels of system energy, fundamental and bifurcated paths of fixed points of the seven first-order differential equations are determined and their stability is investigated. The trajectories of motion of periodically modulated amplitude solutions and their stability are also studied. A model with a higher number of modes is used to evaluate the accuracy of the stability analysis of two-mode nonplanar oscillations perturbed by a two-mode disturbance.

Sommario. Nel presente lavoro si studia la dinamica libera nonlineare di un sistema filo-satellite. L'attenzione è rivolta alle oscillazioni trasversali, governate da due equazioni integro-differenziali, con nonlinearità quadratiche, nelle due componenti di spostamento. Il sistema de debolmente nonlineare ma praticamente lavora in condizioni di risonanza interna. Lo studio è concentrato sui moti nonstazionari generati da perturbazioni delle oscillazioni stazionarie spaziali. Per studiare il problema d stato sviluppato un modello con quattro modi: due sono necessari per descrivere il moto base mentre almeno altri due sono interessati dai fenomeni di risonanza interna quando il moto viene perturbato. Per ottenere le equazioni nelle ampiezze e fasi è stato utilizzato il metodo delle scale multiple. Del sistema di sette equazioni differenziali del primo ordine ottenuto, sono stati studiati i percorsi fondamentali di equilibrio e i rami biforcati, prendendo come parametro il livello di energia totale. È stata inoltre esaminata la stabilità di questi rami. Sono state studiate le traiettorie dei moti periodicamente modulati e la loro stabilità. Infine, è stato utilizzato un modello con un numero più alto di modi per valutare l'accuratezza dell' analisi di stabilità delle oscillazioni bimodali spaziali, nella quale la perturbazione è stata descritta da due soli modi.
\end{abstract}

Key words: String, Nonlinear oscillations, Multiple modal interactions, Motion stability, Bifurcation and chaos

\section{Introduction}

A small satellite connected to an orbiting station by a very long string has recently been used in space research. This has given rise to a large number of papers on the subject in technical journals during the last decade [1-3].

The transversally oscillating orbiting string satellite system exhibits weak nonlinearities in its elongated configuration; these are associated mainly with the longitudinal tension produced by gyroscopic forces, similar to but much smaller than the nonlinear effects of the stretching involved in a taut string with fixed ends. Another effect of gyroscopic forces is the coupling between the longitudinal and transversal motions in the plane of the orbit. The latter element is revealed to be very important because it produces quadratic nonlinearities in 


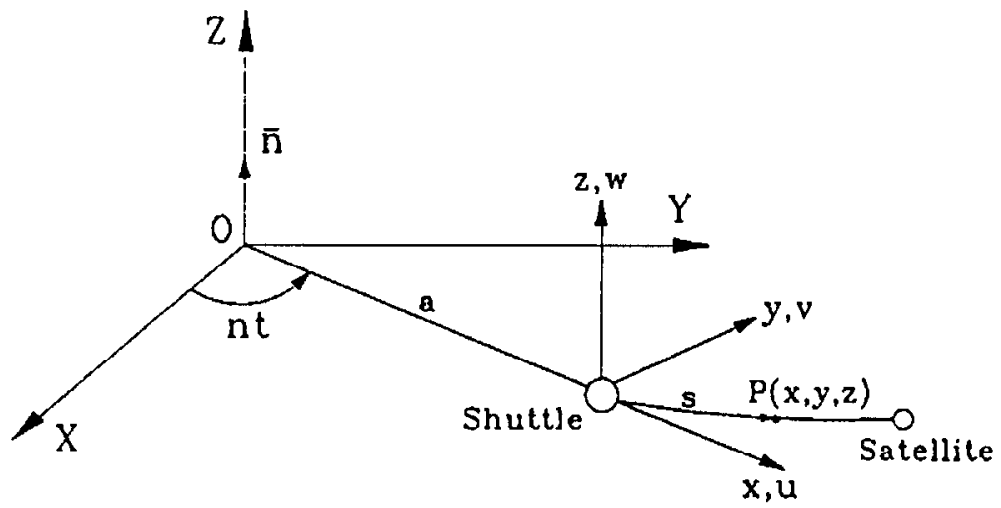

Figure 1. Tethered satellite system and reference frame.

the transversal equations of motion after eliminating the longitudinal displacement through consistent approximation [4-6].

Moreover, apart from the frequency of the almost rigid pendulum-like mode, the frequency spectrum of the deformable modes is virtually an ordered sequence of the type $\omega_{n} \cong n \omega_{1}$ as for taut string. Though weakly nonlinear, the system therefore offers an interesting dynamical behaviour to study due to the occurrence of several simultaneous internal resonances related to its spectrum and the presence of quadratic nonlinearities [7-10]. In a general motion a potentially high number of modes are involved because of the internal resonance phenomenon. However, when a motion with a prevailing modal component is studied, it is possible to analyse the main interaction phenomena with a limited number of modes. Two eigenfunctions associated with the frequencies $\omega_{i}$ and $\omega_{j} \cong 2 \omega_{i}$ are sufficient to describe adequately the planar and nonplanar motion of the string with a prevailing $i$ th modal component. The contribution of higher modes involved by the higher order coupling can be neglected within an approximate solution. In order to analyse the stability of the two-mode oscillations and to find bifurcated solutions, a model with at least three modes must be used to study the out-of-plane perturbed planar motion, which is the subject of a previous work [11].

With the aim of studying stability and nonstationary motions arising from perturbed twomode nonplanar oscillations, a four-mode model is adopted in the present paper, since in this case at least two modes are needed to describe the perturbation of basic oscillation. The multiple time scale method is followed to obtain the amplitude equations. They belong to the class of equations which govern the motion of a four degree-of-freedom Hamiltonian system in 1:2:1:2 simultaneous internal resonances. Fixed points and periodic amplitude oscillations are investigated; bifurcations, linearized stability, and nonstationary motions in the region of unstable solutions are examined. The validity of the mode truncation is discussed by comparing the results obtained using a higher dimension model.

\section{The Equations of Motion and the Discrete Model}

The system under study is a string connected at one end to the shuttle and carrying a satellite at the other end. The system orbits in the XY plane (Figure 1) in equilibrium under centrifugal 
and gravitational forces. Since the mass of the shuttle is much greater than those of the string and the satellite, the centroid of the system coincides in practice with the shuttle; it runs at constant angular velocity $n$ along an orbit assumed to be circular, with radius $a$ related to $n$ through the gravitational constant $\mu_{e}$ by $n^{2}=\mu_{e} / a^{3}$.

An orbiting frame Sxyz, connected to the shuttle, is introduced as shown in Figure 1. A straight reference configuration $x_{0}(s)$ is assumed, where $s$ is the abscissa along the string. The motion is described in this frame by the displacement components $u(s, t), v(s, t), w(s, t)$ measured in relation to configuration $x_{0}(s)$. The equations of motions were obtained in [4] through the Hamilton principle by referring to the elongation as a strain measure.

By developing the displacement components in a Taylor series, three partial differential equations truncated at the third order in the unknowns functions $u(s, t), v(s, t), w(s, t)$ are obtained. In the study of transversal oscillations, bearing in mind that the longitudinal frequency is much higher than the transversal ones, the inertial and centrifugal-gravitational forces can be omitted from the longitudinal equation of motion which furnishes an integro-differential relation among $u, v, w$. By using this relation to eliminate the longitudinal displacement $u(s, t)$, the transversal oscillations of the string-satellite system are governed by two equations in the transversal displacements $[5,6]$.

Let $E A$ be the axial stiffness of the string, $l$ the length, $\mu$ the mass density, $m$ the mass of the satellite. After introducing the dimensionless parameters:

$$
\alpha^{2}=\frac{\mu n^{2} l^{2}}{E A}, \quad \gamma=\frac{\mu l}{m}, \quad \xi=\frac{l}{a},
$$

the dimensionless variables:

$$
\bar{x}_{0}=x_{0} / l, \quad \bar{s}=s / l, \quad \bar{t}=n t, \quad \bar{u}=u / l, \quad \bar{v}=v / l, \quad \bar{w}=w / l
$$

and the dimensionless static tension $f(s)=\left(x_{0}^{\prime}-1\right) / \alpha^{2}$, the following two equations are obtained:

$$
\begin{aligned}
& \ddot{v}-\left[f(s) v^{\prime}\right]^{\prime}-3 \xi x_{0} v-\int_{0}^{s}\left(v^{\prime^{2}}+w^{\prime^{2}}\right) \mathrm{d} s-\left[\frac{2}{\gamma} \dot{v}_{s} v^{\prime}-v^{\prime} \int_{1}^{s} 2 \dot{v} \mathrm{~d} s\right]^{\prime}=0 \\
& \ddot{w}-\left[f(s) w^{\prime}\right]^{\prime}+\left(1-3 \xi x_{0}\right) w-\left[\frac{2}{\gamma} \dot{v}_{s} w^{\prime}-w^{\prime} \int_{1}^{s} 2 \dot{v} \mathrm{~d} s\right]^{\prime}=0
\end{aligned}
$$

with the boundary conditions:

$$
\begin{aligned}
& v(0, t)=w(0, t)=0 \\
& \ddot{v}_{s}+\gamma f(1) v_{s}^{\prime}-3 \xi x_{0}(1) v_{s} \int_{0}^{1}\left(v^{\prime 2}+w^{\prime 2}\right) \mathrm{d} s+2 \dot{v}_{s} v_{s}^{\prime}=0 \\
& \ddot{w}_{s}+\gamma f(1) w_{s}^{\prime}+w_{s}\left(1-3 \xi x_{0}(1)\right)+2 \dot{v}_{s} w_{s}^{\prime}=0,
\end{aligned}
$$

where $v_{s}=v(1, t)$ and $w_{s}=w(1, t)$ are the satellite displacements and the overbar is dropped for the sake of simplicity.

The approximated equations, obtained through a consistent ordering of the linear and nonlinear terms, exhibit only quadratic terms mainly associated witht the gyroscopic forces. The type of nonlinear terms are such that planar solutions in the orbit plane described by the 
displacement $v(s, t)$ can only exist, while the out-of plane component $w(s, t)$ always involves the other component $v(s, t)$ leading to a spatial motion. In view of further developments, equations (3) and (4) are re-written in the integral compact form:

$$
\begin{aligned}
& \left\{M(\ddot{v})+L_{v}(v)+B(v, \dot{v})+C(w, \dot{w})\right\} \cdot \delta v=0 \\
& \left\{M(\ddot{w})+L_{w}(w)+D(w, \dot{v})\right\} \cdot \delta w=01
\end{aligned}
$$

where $M$ and $L$ are linear differential operators of $s$ and $B, C$ and $D$ are bi-linear integrodifferential operators, which are defined in Appendix A.

As is well known [7-10], when quadratic nonlinearities are present the following internal resonance conditions occur: $\omega_{n} \cong 2 \omega_{m}, \omega_{n} \cong\left|\omega_{m} \pm \omega_{k}\right|$, (primary resonances) and $\omega_{n} \cong$ $\omega_{m}, \omega_{n} \cong\left|2 \omega_{m} \pm \omega_{k}\right|, \omega_{n} \cong\left|\omega_{k} \pm \omega_{l} \pm \omega_{m}\right|$ (secondary resonances). The occurrence of multiple modal interactions depends on the sequence of linear frequencies $\omega_{n}$. For the stringsatellite system all the above internal resonance conditions can be verified. Apart the first pendulum mode in the two planes, the linear frequencies of the in-plane and out-of-plane flexible modes practically coincide and both follow the law $\omega_{n} \cong n \omega_{1}$.

Appendix B considers an illustrative example and gives the relevant parameter values. Table 1 shows the sequences of the first frequencies and modes of the system for two string lengths, analytically obtained by assuming a suitable mean value $f_{0}$ for the weakly nonlinearly varying tension $f(s)$ [6]. In particular it is worth noting that the frequencies $\omega_{0}$ of the almost rigid modes are far from the spectrum of the flexible modes and cannot be involved in multiple interaction phenomena.

For the infinite dimensional system an approximate solution can be obtained by a discrete finite model built up by a suitable selection of a limited number of eigenfunctions to describe the deformed configuration of the system. In particular, a nonplanar motion with a prevailing modal component can be adequately represented by two modes, the $k$ th out-of plane mode and the companion $j$ th in-plane mode with frequency $\omega_{j} \cong 2 \omega_{k}$ forced by the quadratic terms $C(w, \dot{w})$ in equation $5_{1}$.

A model able to capture the main bifurcation phenomena must contain other modes in order to describe the perturbation adequately. This may be argued from the variational equations of motion:

$$
\begin{aligned}
& \left\{M(\delta \ddot{v})+L_{v}(\delta v)+B(v, \delta \dot{v})+B(\delta v, \dot{v})+C(w, \delta \dot{w})+C(\delta w, \dot{w})\right\} \cdot \delta v=0 \\
& \left\{M(\delta \ddot{w})+L_{w}(\delta w)+D(w, \delta \dot{v})+D(\delta w, \dot{v})\right\} \cdot \delta w=0 .
\end{aligned}
$$

For an in-plane motion, $w(s, t) \equiv 0$ and equations (6) are uncoupled, enabling the stability for in-plane and out-of-plane disturbances to be studied separately. For spatial motion equations (6) remain coupled and two disturbances $\delta v, \delta w$ must be considered simultaneously.

By describing the disturbance by one in-plane mode of frequency $\omega_{i}$ and one out-ofplane of frequency $\omega_{l}$, these modes must be chosen so that nonlinear terms are resonant in cquation (6). The analysis of resonant terms in the variational equations gives $2 \omega_{i} \cong \omega_{j}$ and $\omega_{l} \cong\left|\omega_{k} \pm \omega_{i}\right|$. When modes in the low frequency spectrum are considered the first meaningful internal resonance condition occurs for $\omega_{i} \cong \omega_{k}, \omega_{l} \cong 2 \omega_{k}$.

Thus in the following a four-mode model, $i$ th and $j$ th in-plane modes and $k$ th and $l$ th outof-plane modes, is used to study nonplanar motion and its stability in the 1:2:1:2 simultaneous internal resonance. 


\section{Amplitude Modulated Equations}

The amplitude modulated equations involving the resonant modes are obtained by the multiple time scale method [7]. The new time scales $t_{n}=\varepsilon_{n} t(n=0,1 \ldots)$ are introduced where $\varepsilon$ is the perturbation parameter; the displacement variables are expanded in a two-term series of $\varepsilon$ :

$$
\begin{aligned}
& v(s, t)=\varepsilon v_{0}\left(s, t_{0}, t_{1}\right)+\varepsilon^{2} v_{1}\left(s, t_{0}, t_{1}\right) \\
& w(s, t)=\varepsilon w_{0}\left(s, t_{0}, t_{1}\right)+\varepsilon^{2} w_{1}\left(s, t_{0}, t_{1}\right) .
\end{aligned}
$$

If equation (7) is substituted in the motion equations, the following perturbation equations will be obtained:

$$
\begin{aligned}
& \left\{M\left(\mathrm{~d}_{00}^{2} v_{0}\right)+L_{v}\left(v_{0}\right)\right\} \cdot \delta v=0 \\
& \left\{M\left(\mathrm{~d}_{00}^{2} w_{0}\right)+L_{w}\left(w_{0}\right)\right\} \cdot \delta w=0 \\
& \left\{M\left(\mathrm{~d}_{00}^{2} v_{1}\right)+L_{v}\left(v_{1}\right)\right\} \cdot \delta v=-\left\{B\left(v_{0}, \mathrm{~d}_{0} v_{0}\right)+C\left(w_{0}, \mathrm{~d}_{0} w_{0}\right)+2 M\left(\mathrm{~d}_{01}^{2} v_{0}\right)\right\} \cdot \delta v \\
& \left\{M\left(\mathrm{~d}_{00}^{2} w_{1}\right)+L_{w}\left(w_{1}\right)\right\} \cdot \delta w=-\left\{D\left(w_{0}, \mathrm{~d}_{0} v_{0}\right)+2 M\left(\mathrm{~d}_{01}^{2} w_{0}\right)\right\} \cdot \delta w,
\end{aligned}
$$

where $\mathrm{d}_{n}=\partial / \partial t_{n}$ and $\mathrm{d}_{m n}^{2}=\partial^{2} / \partial t_{m} \partial t_{n}$.

For the assumed four-mode model the generating solution is described by:

$$
\begin{aligned}
& v_{0}(s, t)=A_{i}\left(t_{1}\right) \varphi_{i}(s) \mathrm{e}^{i \omega_{\imath} t_{0}}+A_{j}\left(t_{1}\right) \varphi_{j}(s) \mathrm{e}^{i \omega_{j} t_{0}}+\text { c.c. } \\
& w_{0}(s, t)=A_{k}\left(t_{1}\right) \psi_{k}(s) \mathrm{e}^{i \omega_{k} t_{0}}+A_{l}\left(t_{1}\right) \psi_{l}(s) \mathrm{e}^{i \omega_{l} t_{0}}+\text { c.c. }
\end{aligned}
$$

where c.c. stands for complex conjugate. The prevailing and companion components of the two-mode nonplanar motion are in primary resonance, while the nonplanar disturbance is in secondary resonance:

$$
\begin{aligned}
& 2 \omega_{i}-\omega_{j}=\varepsilon \sigma_{1} \\
& \omega_{i}+\omega_{k}-\omega_{l}=\varepsilon \sigma_{2} \\
& 2 \omega_{k}-\omega_{j}=\varepsilon \sigma_{3},
\end{aligned}
$$

where $\sigma$ 's are detuning parameters of the order of 1 .

Equations (10) are substituted in equations (9) and by zeroing secular terms, taking account of equations (11), four ODEs in the complex amplitude $A_{i}, A_{j}, A_{k}$ and $A_{l}$ are obtained:

$$
\begin{aligned}
& A_{i}^{\prime}+2 k_{1} \bar{A}_{i} A_{j} \mathrm{e}^{-i \sigma_{1} t_{1}}-2 k_{2} A_{l} \bar{A}_{k} \mathrm{e}^{-i \sigma_{2} t_{1}}=0 \\
& A_{j}^{\prime}+2 k_{3} A_{i}^{2} \mathrm{e}^{i \sigma_{1} t_{1}}+2 k_{4} A_{k}^{2} \mathrm{e}^{i \sigma_{3} t_{1}}=0 \\
& A_{k}^{\prime}-2 k_{5} A_{l} \bar{A}_{i} \mathrm{e}^{-i \sigma_{2} t_{l}}-2 k_{6} \bar{A}_{k} A_{j} \mathrm{e}^{-i \sigma_{3} t_{1}}=0 \\
& A_{l}^{\prime}+2 k_{7} A_{k} A_{i} \mathrm{e}^{i \sigma_{2} t_{1}}=0,
\end{aligned}
$$

where the overbar denotes complex conjugate, prime $t_{1}$-differentiation and the coefficients $k_{i}$ are defined in Appendix C. By introducing the polar form:

$$
A_{n}=\frac{1}{2} a_{n}\left(t_{1}\right) \mathrm{e}^{i \theta_{n}\left(t_{1}\right)} \quad(n=i, j, k, l)
$$


and separating real and imaginary parts in equations (12), eight equations in the four real amplitudes $a_{n}$ and four phases $\theta_{n}$ are obtained.

The use of the new variables:

$$
\begin{aligned}
\gamma_{1} & =2 \vartheta_{\imath}-\vartheta_{j}+\sigma_{1} t_{1} \\
\gamma_{2} & =\vartheta_{i}+\vartheta_{k}-\vartheta_{l}+\sigma_{2} t_{1} \\
\gamma_{3} & =2 \vartheta_{k}-\vartheta_{j}+\sigma_{3} t_{1}
\end{aligned}
$$

makes it possible to reduce the previous system to an autonomous system of seven equations in the state variables $z=\left(a_{i}, a_{j}, a_{k}, a_{l}, \gamma_{1}, \gamma_{2}, \gamma_{3}\right)^{T}$. They read:

$$
\begin{aligned}
& a_{i}^{\prime}=-k_{1} a_{i} a_{j} \cos \gamma_{1}+k_{2} a_{l} a_{k} \cos \gamma_{2} \\
& a_{j}^{\prime}=k_{3} a_{i}^{2} \cos \gamma_{1}-k_{4} a_{k}^{2} \cos \gamma_{3} \\
& a_{k}^{\prime}=k_{5} a_{l} a_{i} \cos \gamma_{2}+k_{6} a_{k} a_{j} \cos \gamma_{3} \\
& a_{l}^{\prime}=-k_{7} a_{k} a_{i} \cos \gamma_{2} \\
& a_{i} a_{j} \gamma_{1}^{\prime}=\left(2 k_{1} a_{i} a_{j}^{2}-k_{3} a_{i}^{3}\right) \sin \gamma_{1}-2 k_{2} a_{l} a_{k} \sin \gamma_{2}+k_{4} a_{i} a_{k}^{2} \sin \gamma_{3}+\sigma_{1} a_{i} a_{j} \\
& a_{i} a_{k} a_{l} \gamma_{2}^{\prime}=k_{1} a_{i} a_{j} a_{k} a_{l} \sin \gamma_{1}+\left(k_{7} a_{i}^{2} a_{k}^{2}-k_{2} a_{k}^{2} a_{l}^{2}-k_{5} a_{i}^{2} a_{l}^{2}\right) \sin \gamma_{2} \\
& \quad-k_{6} a_{i} a_{j} a_{k} a_{l} \sin \gamma_{3}+\sigma_{2} a_{i} a_{k} a_{l} \\
& a_{j} a_{k} \gamma_{3}^{\prime}=-k_{3} a_{i}^{2} a_{k} \sin \gamma_{1}-2 k_{5} a_{i} a_{j} a_{l} \sin \gamma_{2}+\left(k_{4} a_{k}^{3}-2 k_{6} a_{j}^{2} a_{k}\right) \sin \gamma_{3}+\sigma_{3} a_{j} a_{k} .
\end{aligned}
$$

A suitable combination of the first four equations furnishes a first integral of motion which states the conservation of energy:

$$
a_{i}^{2}+\beta_{j}^{2} a_{j}^{2}+\beta_{k}^{2} a_{k}^{2}+\beta_{l}^{2} a_{l}^{2}=E
$$

where:

$$
\beta_{j}^{2}=\frac{k_{1}}{k_{3}}, \quad \beta_{k}^{2}=\frac{k_{1}}{k_{3}} \frac{k_{4}}{k_{6}}, \quad \beta_{l}^{2}=\left(k_{2}+\frac{k_{1}}{k_{3}} \frac{k_{4}}{k_{6}} k_{5}\right) / k_{7} .
$$

This entails that the trajectories lie on a ipersphere in the space of the normalized amplitudes: $\tilde{a}_{i}=a_{i}, \tilde{a}_{j}=\beta_{j} a_{j}, \tilde{a}_{k}=\beta_{k} a_{k}, \tilde{a}_{l}=\beta_{l} a_{l}$.

\section{Steady-state and Periodic Amplitude Solutions}

The steady-state solutions are the fixed points of equations (15). These equations admit solutions with one, two or four non-vanishing components only; ariong these attention is focused on the non-planar oscillations described by $a_{k} \neq 0, a_{j} \neq 0$ and $a_{i}=a_{l}=0$.

The two-mode amplitude modulated oscillations are governed by three of equations (15) in which $a_{i}=a_{l}=0$ :

$$
\begin{aligned}
& a_{k}^{\prime}=k_{6} a_{k} a_{j} \cos \gamma_{3} \\
& a_{j}^{\prime}=-k_{4} a_{k}^{2} \cos \gamma_{3} \\
& a_{j} a_{k} \gamma_{3}^{\prime}=\left(k_{4} a_{k}^{3}-2 k_{6} a_{j}^{2} a_{k}\right) \sin \gamma_{3}+\sigma_{3} a_{j} a_{k} .
\end{aligned}
$$




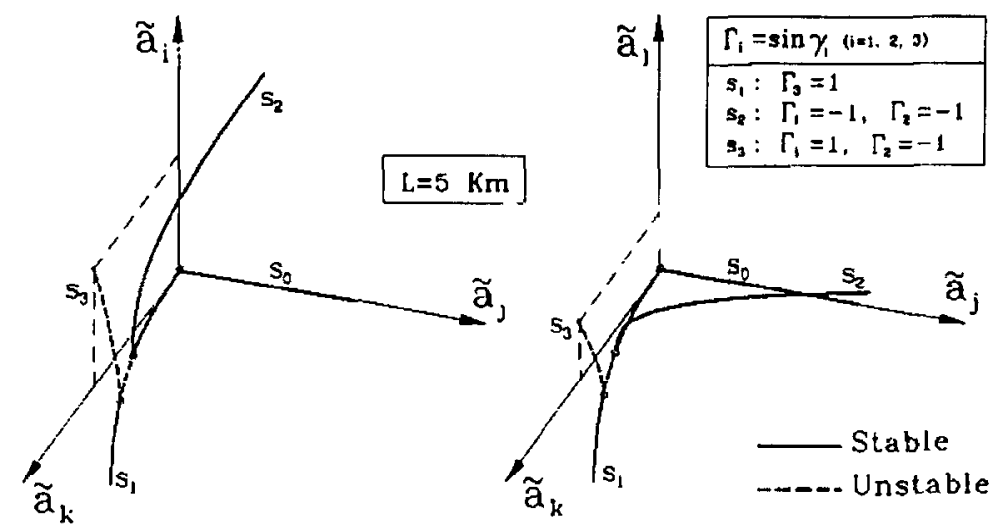

Figure 2. Amplitude equilibrium paths for $k$ th and $\jmath$ th primary resonant modes and bifurcated paths for $i$ th and $l$ th disturbance modes.
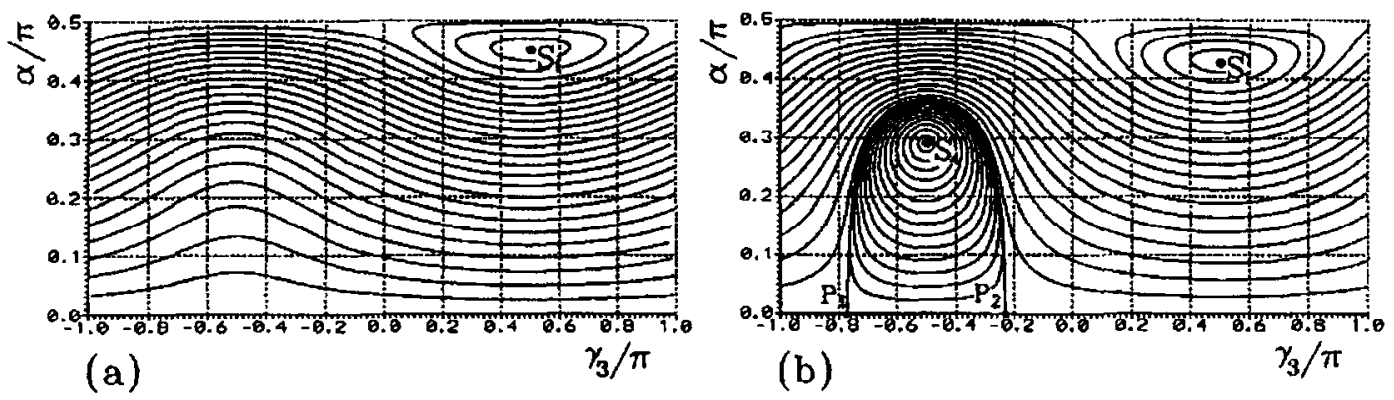

Figure 3. Steady-state and periodic solutions in the space $\left(\gamma_{3}, \alpha\right)$ with $\tan \alpha=\tilde{a}_{k} / \tilde{a}_{\jmath}$, for (a) lower and (b) higher levels of energy.

The steady-state solutions are obtained by zeroing the right-hand side terms of equation (18) with the following result:

$$
\begin{aligned}
& \gamma_{3}= \pm \frac{\pi}{2} \\
& \pm\left(k_{4} a_{k}^{2}-2 k_{6} a_{j}^{2}\right)+\sigma_{3} a_{j}=0 .
\end{aligned}
$$

The curve $a_{k}-a_{j}$ is represented in Figure 2 for the sample system oscillating with the prevailing out-of-plane first mode ( $k=1, j=2$ ) perturbed by the prevailing in-plane mode $(i=1, l=2$ ) in secondary resonance. On the two-mode curves a bifurcation analysis for a non-planar disturbance $a_{i}-a_{l}$ is developed by means of the procedure explained in the next Section. For the curve $s_{1}$, depending on the system parameters, two points of bifurcation occur for $\sin \gamma_{1}= \pm 1, \sin \gamma_{2}=-1$.

The equilibrium paths $s_{2}$ and $s_{3}$ with four non-vanishing components are numerically determined, starting from the bifurcation points (Figure 2). On these curves constant values of $\gamma_{i}$ are obtained, $\gamma_{1}= \pm \pi / 2, \gamma_{2}=-\pi / 2$ and $\gamma_{3}=\pi / 2$, which is an extension of what happens on the two-mode curve. For the bifurcated four-mode solutions no saturation phenomena occur, as in planar oscillation perturbed out-of-plane in secondary resonance [11]. 
Different representations of the solutions of equations (19) have been given in the literature; those in $[7,12]$ are very effective. Due to the existence of the first integral of motion (16), for an energy level $E$ the trajectories lie on a cylinder. The trajectories numerically obtained from equations (18) are therefore presented on the plane $\alpha-\gamma_{3}$ where $\tan \alpha=\tilde{a}_{k} / \tilde{a}_{j}$ (Figure 3).

Two different levels of energy are illustrated in Figure 3 where $\gamma_{3}$ varies in $[-\pi, \pi]$. For a low level (Figure 3(a)) one stationary two-mode solution $S_{1}$ at $\gamma_{3}=\pi / 2$ and onemode solution path $s_{0}\left(a_{k}=0, \gamma_{3}\right.$ arbitrary) occur, according to the paths in Figure 2. Both solutions are stable. Weak amplitude modulation occurs on each trajectory, while strong phase modulation occurs on most of them, except for the closed trajectories that fill a small region around $S_{1}$.

For a higher value of energy (Figure 3(b)) a new stationary solution $S_{4}$ appears. Since, in contrast to the planar case, it occurs for a very high energy level, the curve $s_{4}$ is not reported in Figure 2. Another region of closed trajectories is generated, enclosed by a separatrix joining the two singular points $P_{1}$ and $P_{2}$ which make the in-plane oscillation $\alpha=0$ become unstable. Points $P_{1}$ and $P_{2}$ are located at $\gamma_{3}=\arcsin \left(\sigma_{3} /\left(2 k_{6} a_{j}\right)\right)$ obtained from the right-hand side term of equation (183) equated to zero for vanishing $a_{k}$. They thus depend on the energy, while the tangents to the separatrix in $P_{1}$ and $P_{2}$ are always vertical, in contrast to a similar case illustrated in [13] where the position of the bifurcation points is fixed but the tangent depends on the energy. In the case of Figure 3(b) a strong modulation of amplitude and phase occurs on some trajectories.

To obtain the displacements $v(s, t)$ and $w(s, t)$, according to equations (10) and (13), the $\theta_{k}$ and $\theta_{j}$ are evaluated from:

$$
\begin{aligned}
& \vartheta_{k}^{\prime}=-k_{6} a_{j} \sin \gamma_{3} \\
& \vartheta_{j}^{\prime}=-k_{4} \frac{a_{k}^{2}}{a_{j}} \sin \gamma_{3}
\end{aligned}
$$

which are the imaginary parts of equations (12) when $A_{i}=A_{l}=0$. Since the right-hand side terms of equation (20) are $T$-periodic, denoting by $\nu_{n}$ their mean value, $\theta_{n}$ can be written as:

$$
\vartheta_{n}(t)=\nu_{n} t+\chi_{n}(t), \quad n=k, j
$$

where $\nu_{n}$ is the nonlinear modification of the mean instantaneous frequency $\omega_{n}$ and $\chi_{n}(t)$ is $T$-periodic function, accounting for the phase modulation. By substituting equation (21) into equation $\left(14_{3}\right)$, taking into account that $\gamma_{3}$ and $\chi_{n}$ are periodic, the non-periodic terms must vanish:

$$
2 \nu_{k}-\nu_{j}+\sigma_{3}=0
$$

The displacement is thus:

$$
\begin{aligned}
& v(s, t)=a_{j}(t) \varphi_{j}(s) \cos \left[2 \Omega_{k} t+\chi_{j}(t)\right] \\
& w(s, t)=a_{k}(t) \psi_{k}(s) \cos \left[\Omega_{k}+\chi_{k}(t)\right],
\end{aligned}
$$

where $\Omega_{k}=\omega_{k}+\nu_{k}$ is the nonlinear frequency and $\Omega_{j}=\omega_{j}+\nu_{j}=2 \Omega_{k}$ follows from equations $\left(11_{3}\right)$ and $(22)$. Nonlinearities adjust the frequencies of the two modes in the ratio $1: 2$ as in the stationary case. However, strictly speaking the displacement (23) describes 

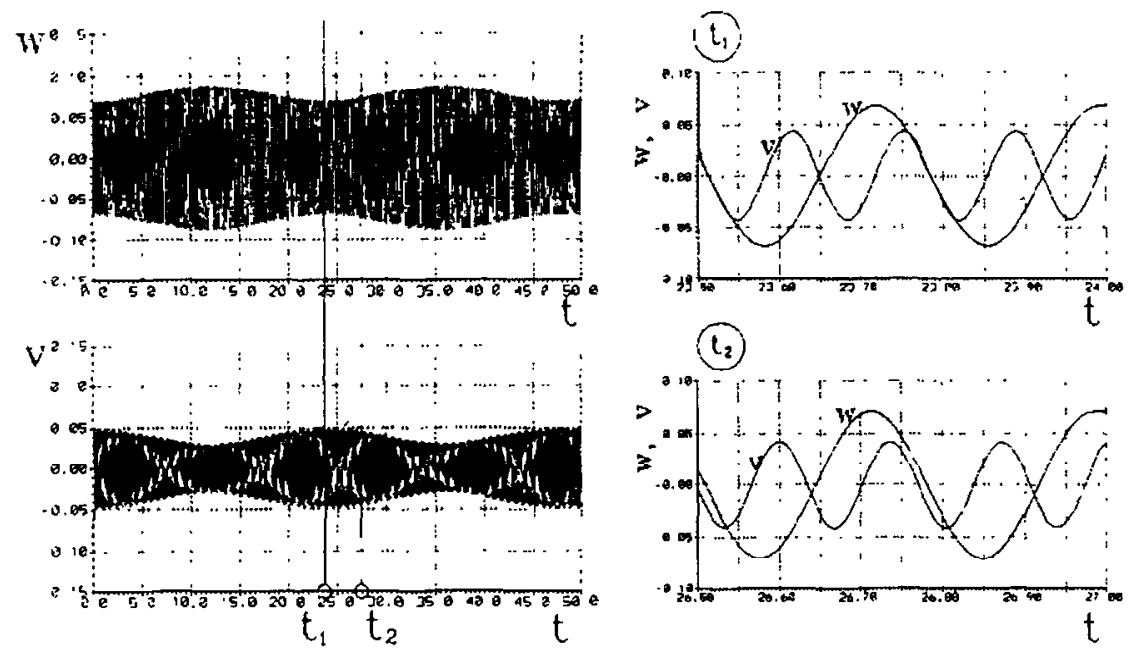

(t)

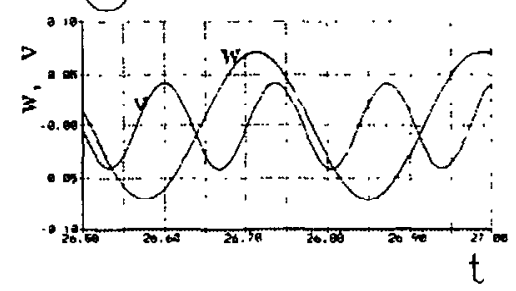

Figure 4. Time history of the displacement components $w$ and $v$ at $s=1 / 4$ ( $t_{1}$ minimum drift, $t_{2}$ maximum drift).
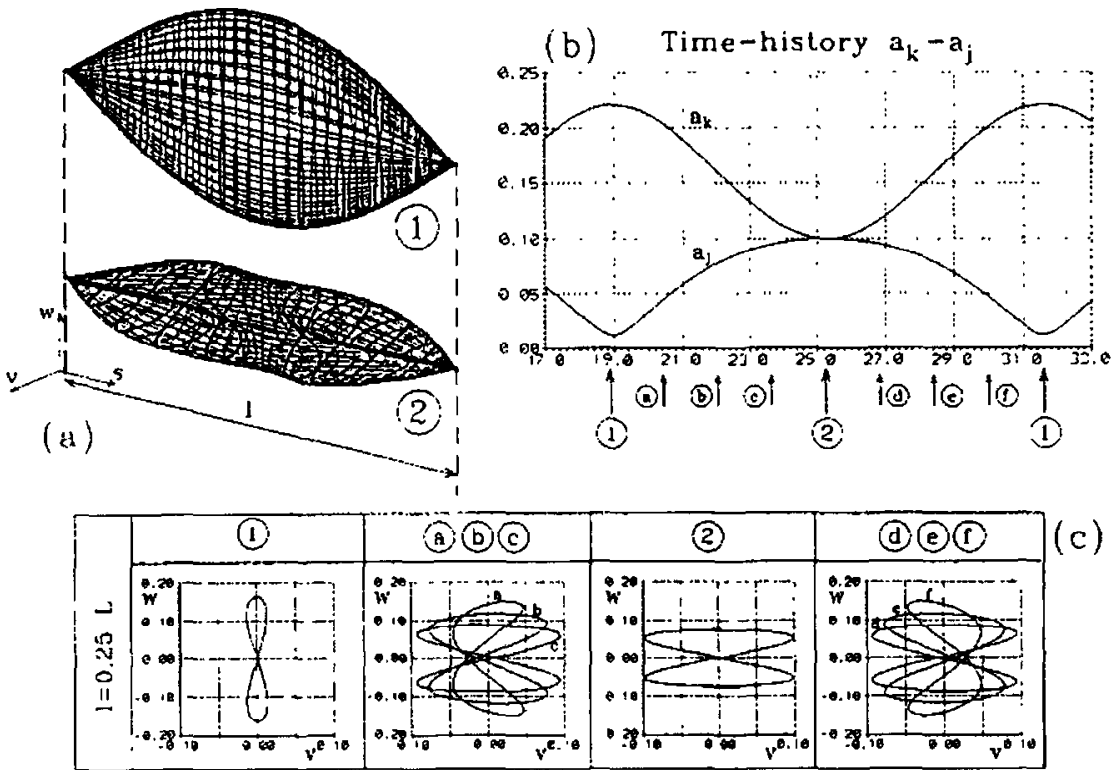

Figure 5. Evolution of the deformed configuration in a fast period for some instants between those corresponding to zero drift (1) to (2)).

an almost periodic oscillation because the period $T$ of the slow modulation is in general incommensurable with the period of fast oscillation. In the steady oscillations $\chi_{n}(t)$ and $a_{n}(t)$ are constant.

The amplitude modulated displacements (23) at $s=\frac{1}{4}$ are plotted in Figure 4 for the string oscillating in the first prevailing out-of-plane mode $(k=1)$ with the second companion 
in-plane mode $(j=2)$. Each component shows an amplitude modulation associated with the internal resonance phenomenon but the shape generated by their contributions is strongly governed by the phase difference between them. The minimum and maximum values of these differences can be appreciated from the zoomed time-histories in Figure 4, starting at two different instants, $t_{1}$ and $t_{2}$, respectively.

The oscillation shape changes during a period due to the contributions of the two modes oscillating with multiple frequencies. The picture of the deformed configuration in one fast period is shown in Figure 5(a) around the instants marked in Figure 5(b); the trajectories described by the material point of the string at $s=\frac{1}{4}$ are drawn in Figure $5(\mathrm{c})$. The instants $t_{1}$ and $t_{2}$ correspond to zero drift conditions.

\section{Stability of Steady-state and Periodic Amplitude Oscillations}

The stability analysis of the four-mode fixed point solutions can be performed straightforwardly by means of the variational equations by determining the eigenvalues of the Jacobian evaluated at points of the curve. The results of the analysis are shown in Figure 2, in which the stable (unstable) branches are solid (broken) lines. The stability of the steady-state two-mode solutions cannot be analysed in the same way because the amplitude equations cannot be written in normal form, due to $a_{i}=a_{l}=0$. A Cartesian representation of complex amplitudes must be used, as in $[14,15]$; however, a more general procedure, suitable for analysing the stability of periodic amplitude solutions, has been followed here.

The amplitude equations (12) are non-autonomous; to render them autonomous the new amplitudes $Z_{n}$ are introduced:

$$
A_{n}=Z_{n} \mathrm{e}^{i \alpha_{n} t_{1}}, \quad n=i, j, k, l \text {. }
$$

The constants $\alpha_{k}, \alpha_{j}$ related to the non-vanishing amplitudes of the two-mode periodic solutions $a_{k_{0}}(t), a_{j_{0}}(t), \gamma_{3_{0}}(t)$, are taken to be equal to the frequency corrections, $\alpha_{k}=$ $\nu_{k_{0}}$ and $\alpha_{j}=\nu_{j_{0}}$. This produces a first important result: while the complex amplitudes $A_{n_{0}}=\frac{1}{2} a_{n_{0}}(t) \exp \left(i \vartheta_{n_{0}}(t)\right)$ are not periodic due to the form (21) of $\vartheta_{n_{0}}(t)$, the amplitudes $Z_{n_{0}}(t)=\frac{1}{2} a_{n_{0}}(t) \exp \left(i \chi_{n_{0}}(t)\right)(n=k, j)$ are periodic since $\chi_{n_{0}}$ and $a_{n_{0}}$ are periodic.

Moreover, this choice of $\alpha_{k}, \alpha_{j}$ zeroes some non-autonomous terms, while the rest vanish when $\alpha_{i}, \alpha_{l}$ satisfy the conditions:

$$
\begin{aligned}
& 2 \alpha_{i}-\alpha_{j}+\sigma_{1}=0 \\
& \alpha_{i}+\alpha_{k}-\alpha_{l}+\sigma_{2}=0 .
\end{aligned}
$$

The new system is:

$$
\begin{aligned}
& Z_{i}^{\prime}+2 k_{1} \bar{Z}_{i} Z_{j}+2 k_{2} Z_{l} \bar{Z}_{k}+i \alpha_{i} Z_{i}=0 \\
& Z_{j}^{\prime}-2 k_{3} Z_{i}^{2}+2 k_{4} Z_{k}^{2}+i \alpha_{j} Z_{j}=0 \\
& Z_{k}^{\prime}+2 k_{5} \bar{Z}_{i} Z_{l}-2 k_{6} Z_{j} \bar{Z}_{k}+i \alpha_{k} Z_{k}=0 \\
& Z_{l}^{\prime}+2 C_{l} Z_{i} Z_{k}+i \alpha_{l} Z_{l}=0 .
\end{aligned}
$$

Thus the introduction of $Z_{n}$ variables makes it possible to reconduct the stability analysis of the periodic amplitude motion to the solution of a standard variational problem with periodic 

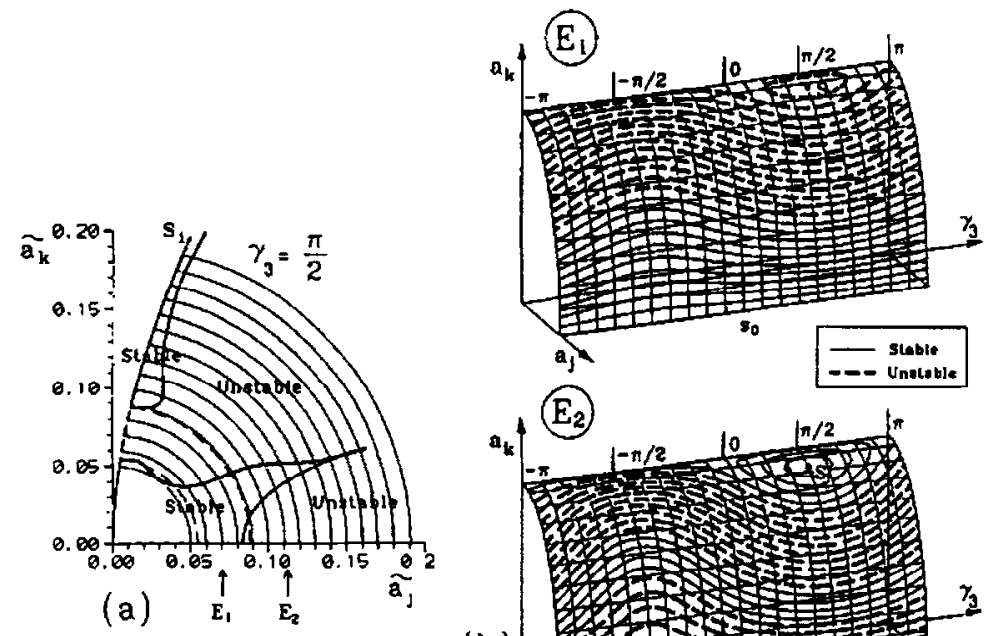

(b)

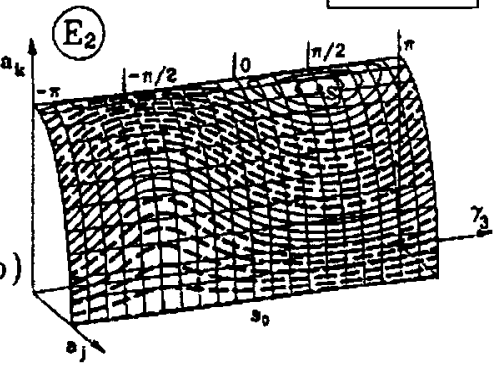

Figure 6. (a) Instability regions of the trajectories on the section at $\gamma_{3}=\pi / 2$ in the $\left(\tilde{a}_{k}, \tilde{a}_{j}, \gamma_{3}\right)$ space and (b) stable (- ) and unstable (- --- ) nonplanar periodic amplitude oscillations for the two levels of energy $E_{1}$ and $E_{2}$.

coefficients. In particular, in the simpler case of steady motions where $a_{n_{0}}$ and $\chi_{n_{0}}$ are constant, the procedure adopted here is reduced to that followed in $[14,15]$.

The stability of the two-mode oscillations perturbed by the new components, $i$ th and $l$ th, is described by the sub-system of variational equations, involving the only perturbed components:

$$
\begin{aligned}
\delta Z_{i}^{\prime} & =-\left(2 k_{1} Z_{j 0} \delta \bar{Z}_{i}+2 k_{2} \bar{Z}_{k_{0}} \delta Z_{l}+i \alpha_{i} \delta Z_{i}\right) \\
\delta Z_{l}^{\prime} & =-\left(2 k_{7} Z_{k_{0}} \delta Z_{i}+i \alpha_{l} \delta Z_{l}\right) .
\end{aligned}
$$

After introducing the Cartesian form for $Z_{n_{0}}(n=k, j)$, the stability of the periodic nonplanar motion is analysed by means of the eigenvalues of the monodromy matrix determined by the numerical integration of equation (27).

In the particular case of steady oscillations, eigenvalues such as $\lambda= \pm \sqrt{c}$, with $c$ real constant, are found; at the critical condition the two eigenvalues coalesce in the origin of the complex plane. In the periodic case the eigenvalues run along the unitary circle and coalesce in $\lambda=1$ at the critical condition.

In Figure 6(a) the stability regions on a section of state space are reported for the illustrative example. The most interesting aspect is the occurrence of two close bifurcation points on the stationary branch $s_{1}$ which makes the steady oscillations lose and regain their stability. Moreover, it can be appreciated that for low levels of energy almost all oscillations are stable up to the level of the first bifurcation; between the first and second bifurcation, the steady solutions and a certain close region of orbits are unstable while the furthest are stable; above the last bifurcation the steady solutions and a region of periodic orbits, near the steady solutions, regain stability while the one-mode solutions $a_{j} \neq 0, a_{i}=a_{k}=a_{l}=0$ and periodic 

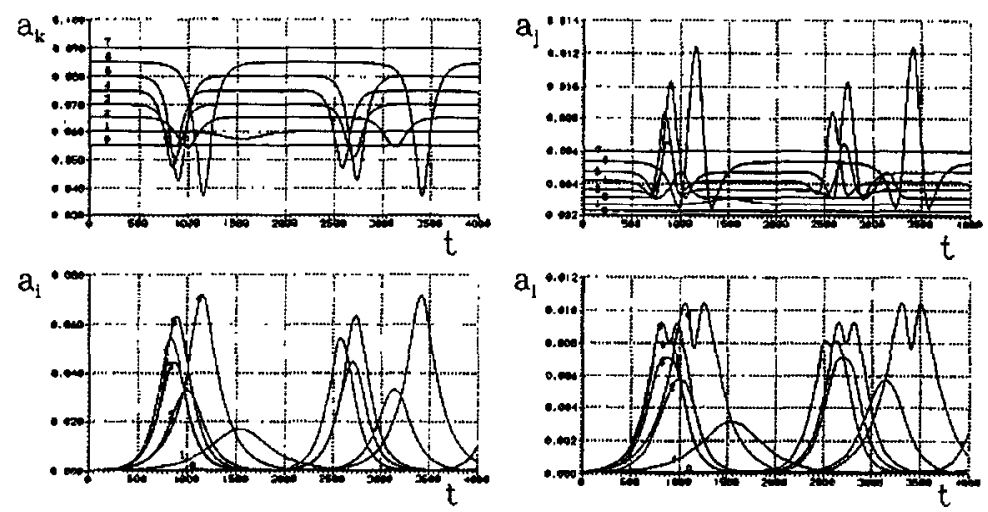

Figure 7. Amplitude time histories for nonplanar steady-state solutions $(k=1, j=2)$ with increasing energy perturbed by secondary resonant modes $(i=1, l=2)$. Cases 0 and 7 correspond to bifurcation points.

(1)

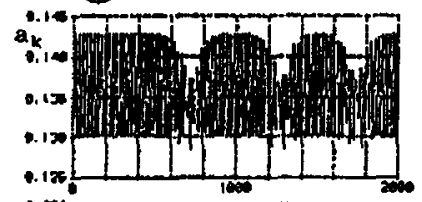

a.eof

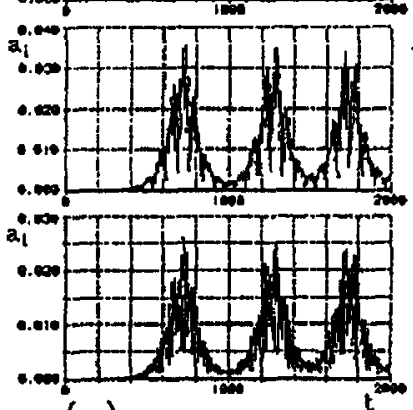

(a)
(4)
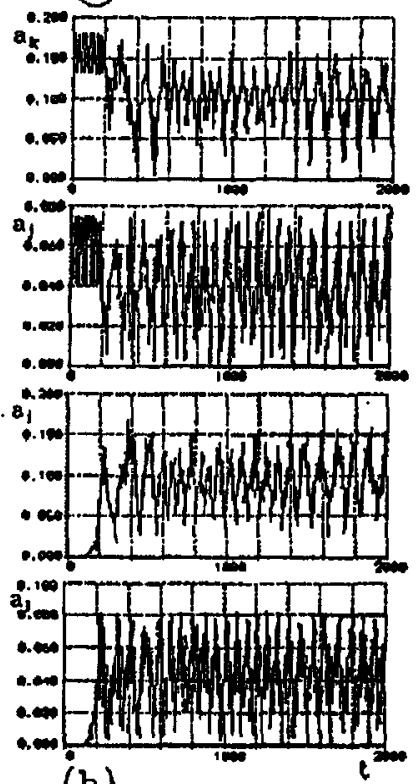

(b)

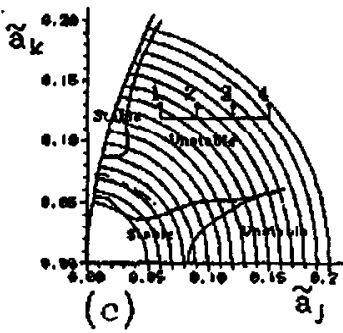

Figure 8. Amplitude time-histories for two cases ( $\mathrm{a}$ and $\mathrm{b}$ ) of nonplanar periodic solutions $(k=1, j=2)$ with increasing energy (c) perturbed by secondary resonant modes $(i=1, l=2)$.

orbits near the previous lose stability. Figure $6(\mathrm{~b})$ reports stable and unstable trajectories and oscillations for the two levels of energy $E_{1}$ and $E_{2}$ marked in Figure 6(a).

\section{Numerical Investigation of Four-mode Nonstationary Motions}

Some numerical results concerning the evolution of the state variables for some unstable steady and periodic oscillations of the two-mode $(k-j)$ non-planar motion perturbed by non-planar disturbance $(i-l)$ are presented. The solutions are obtained by integrating the amplitude equations (15). 

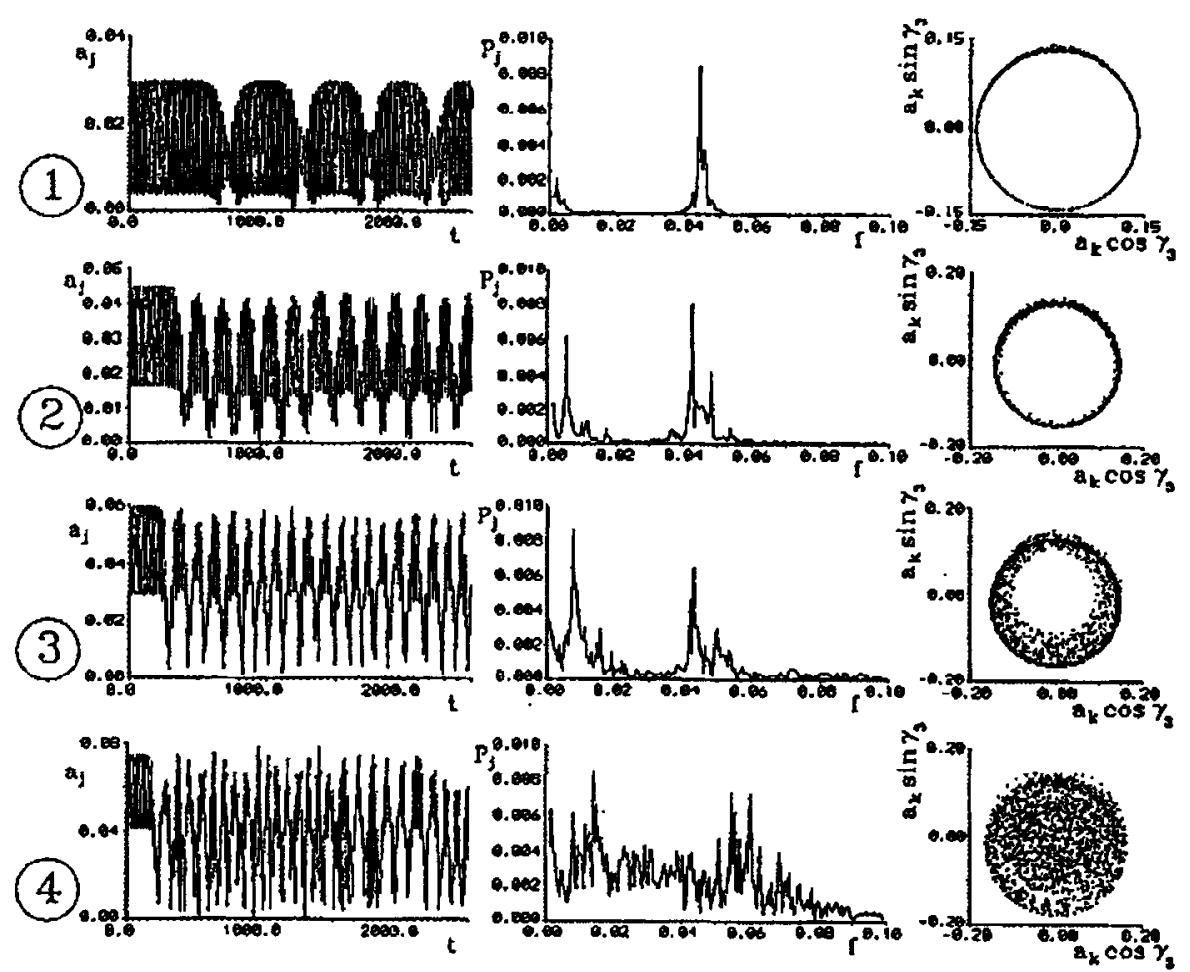

Figure 9. The phase-plane trajectory of component $a_{k}$, the time-history and the frequency content of the evolution of component $a$, of the perturbed two-mode oscillations for the four cases (1-4) reported in Figure 8(c).

It is useful to examine first the behaviour of perturbed steady solutions whose stable and unstable branches have already been shown in Figure 2. Unstable equilibrium points on the branch $s_{1}$, starting from the bifurcation points, are perturbed by the same small perturbance $a_{\imath}(0), a_{l}(0)$ and the evolution of the four amplitudes is drawn in Figure 7 . The steady motion is transformed into a periodic motion with a very regular exchange of energy from the $k, j$ modes to the $i, l$ perturbance modes.

As the energy of the oscillation increases from the first (curve 0 ) to the second (curve 7 ) bifurcation point, the period of the amplitude modulation initially decreases and subsequently increases steadily, changing its behaviour practically in the middle of the unstable branch, while the energy transfer always increases up to the second bifurcation point, where it quickly vanishes. The energy transfer among the prevailing components of the basic and disturbance modes is very regular, i.e. while $a_{k}$ decreases $a_{i}$ increases. The transfer of energy between the companion components is more complex at the highest energy levels while remaining periodic.

Unlike the behaviour of the planar two-mode oscillations out-of-plane perturbed by one mode disturbance [11], only the periodically amplitude modulated oscillations close to the boundaries of the stable regions evolve in a regular way when perturbed. Four orbits with increasing energy levels and progressively farther from the stable region are considered. The time histories of the amplitudes for the two extreme cases 1 and 4 are reported in Figure 8 . In the first regular case the energy transfer towards the perturbed components concerns the mean valuc of amplitudes while remaining the modulation. In case 4 this transfer is irregular, giving rise to a very irregular motion. 


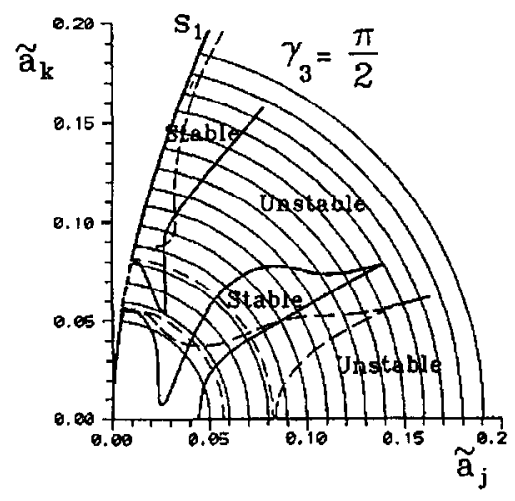

Figure 10. Instability regions of the trajectories on the section at $\gamma_{3}=\pi / 2$ in the $\left(\tilde{a}_{k}, \tilde{a}_{3}, \gamma_{3}\right)$ space for the 6 d.o.f. model ( $\longrightarrow$ ) and for the 4 d.o.f. model $(----)$.

To understand the transition from regular to irregular motions better, the four cases $(1-4)$ are illustrated in Figure 9. For each case three meaningful portraits are given: the phase-plane trajectory of the prevailing component $a_{k}$ sampled at constant step $\Delta t$, the time-history and the relevant frequency content of the companion component $a_{j}$. Case (1) is very regular; the frequency spectrum shows two groups of a few peaks around two frequencies, the lower associated with the slow energy transfer among the two components of the unstable basic oscillations and the other two perturbed and the higher frequency related to the fast modulation of the unperturbed orbit. As the energy level increases, the period of the energy transfer becomes shorter and both groups become wider due to the modification of time laws and the occurrence of new harmonics (cases 2 and 3 ). This can be appreciated on the phase plane where the trajectories describe a bundle. When these two groups overlap, an explosion is observed and the phenomenon appears completely irregular (case 4).

\section{Remarks on the Truncated Model for the Stability Analysis}

In the stability analysis of the two-mode oscillations dealt with previously, the disturbance has been described by two modes. This truncation of the model furnishes approximate results. In order to evaluate the accuracy of the results obtained, a higher number of modes is used to represent the disturbance in this Section.

The $r$ th planar mode with frequency $\omega_{r} \cong 3 \omega_{k}$ and the $s$ th out-of-plane mode with $\omega_{s} \cong 4 \omega_{k}$ are added; the six-degree-of-freedom model obtained again admits the two-mode $a_{k}-a_{j}$ solution previously considered and the other four modes represent all the resonant modes in the variational equations (6). Here, this enlarged model is used only to investigate the boundaries of the stability regions, without analysing the evolution of the unstable motions.

In order to obtain the variational equations that govern the evolution of the disturbance amplitude, the procedure already followed is employed and the relevant equations are reported in Appendix D. The governing solution now contains the six selected modes (D.1) and two new internal resonance conditions (D.2) involving the $\omega_{r}, \omega_{s}$ frequencies are added to those of equations (11). Six first-order differential equations in the complex amplitudes $A_{n}$ are obtained (D.3). A change in the variables $A_{n}=Z_{n} \mathrm{e}^{i \alpha_{n} t_{1}}$ leads to an autonomous system (D.4) when the coefficients $\alpha_{n}$ satisfy equation (25) and the new conditions (D.5). 
Finally, the stability of the two-mode oscillations is governed by four equation systems (D.6) in the disturbance components $\delta Z_{n}(n=i, l, r, s)$ which are uncoupled from the others. The analysis of the eigenvalues of the monodromy matrix and the Jacobian matrix define the stability of the periodic and steady state $a_{k}-a_{j}$ oscillations respectively.

In Figure 10 the new boundaries (solid line) of the stability regions are reported together with those (dashed line) already reported in Figure 6. It is apparent that the enlargement of the assumed modes does not substantially affect the scenario; however, since the shape of the region is modified a wider variety of stable or unstable oscillations can be found for an assigned energy level depending on the initial conditions. In particular, some modulated orbits corresponding to a much smaller energy level than that of the first bifurcation point are now found to be unstable. The bifurcation points on the steady-state two-mode path $\left(a_{k} \neq 0, a_{j} \neq 0\right)$ remain practically unchanged, while on the one-mode path $\left(a_{j} \neq 0\right)$ the critical amplitude notably decreases.

\section{Conclusions}

After condensing the longitudinal displacement, the nonlinear transversal oscillations of a tethered satellite system can be adequately described by two equations. These reveal weak quadratic nonlinear terms, due mainly to gyroscopic forces, and several conditions of internal resonance due to the dynamic characteristic of the system. In order to study nonplanar oscillations, a two-mode model can accurately describe nonplanar oscillations with a prevailing $k$ th mode and companion $j$ th mode with frequency $\omega_{j} \cong 2 \omega_{k}$. Richer models are needed to study more complex motions. In particular, a four-mode model is used to analyse bifurcation, stability and the evolution of unstable nonplanar motions, since in this case the perturbation of basic oscillation requires at least two modes to describe it.

The stability of the steady and periodic solutions are analysed by introducing a Cartesian representation of complex amplitudes, since the amplitude equations cannot be written in normal form. By means of a suitable transformation of the complex amplitudes, the stability of periodic motions is governed by a system of variational equations with periodic coefficients. Two close bifurcation points are found on the stationary branch, which makes the steady oscillations lose and regain stability. Periodic amplitude oscillations are stable for low levels of energy; between the energy level of the first and second bifurcations, a certain region of orbits is unstable while the further orbits are stable. Beyond the last bifurcation a region of periodic orbits, near the steady solutions, regains stability, while the orbits near the one-mode solution $a_{j} \neq 0, a_{i}=a_{k}=a_{l}=0$ become unstable.

The numerical solution of the seven-dimensional system of the amplitude equations furnishes a description of the nonstationary four-mode motions in conditions of simultaneous internal resonances. Unstable steady two-mode solutions perturbed by small disturbances are trasformed into periodic four-mode motions that are slowly modulated with a very regular exchange of energy from the basic modes to the perturbance modes. For nonplanar periodic oscillations perturbed by a small two-mode disturbance, only the periodically amplitude modulated oscillations close to the boundaries of the stable regions evolve in a regular way when perturbed. In this case the amplitude modulations of the four components remain, but they occur around a mean value which is modulated on a slower scale. In other cases the transfer of energy is irregular, giving rise to noticeably irregular motions; some representative cases are illustrated by the time-history, phase-plane and frequency content of components. 
In order to evaluate the accuracy of the four-mode model in stability analysis, a richer model is used in which the disturbance is represented by four modes. The enlargement of the assumed modes does not susbtantially affect the scenario but the modification of the shape of the region produces a wider variety of stable and unstable oscillations. The bifurcations points on the steady-state solution path remain practically unchanged.

\section{Acknowledgement}

This work was partially supported by Ministry of University and Scientific and Technological Research (MURST-60\%, 1994).

\section{References}

1. Banerjee, A.K. and Kane, T.R., 'Tether development dynamics', J. Astronautical Sciences, 30 (1982) $347-$ 365.

2. Misra, A.D. and Modi, V.J., 'A survey on the dynamics and control of tethered satellite system'. In: Proceedings of the NASA/AIAA/PSN Incernational Conference on Tethers in Space, 1986, pp. 667-719.

3. von Flotow, A.H., 'Some approximations for the dynamics of tethered spacecraft', J. Guidance, Control and Dynamics, 11 (1988) 357-364.

4. Pasca, M., Luongo, A., Pignataro, M. and Vestroni, F., Free Dynamics of the Shuttle - Tethered-Satellite System, Report No. 3, Dpt. of Structural and Geotechnical Engineering, University of Rome 'La Sapienza', Rome, Italy, 1987.

5. Vestroni, F. and Luongo, A., 'Perturbation analysis of finite oscillations of an orbiting string'. In: W. Schneider, H. Troger, F. Zeigler (eds), Trends in Applications of Mathematics to Mechanics, Longman Scientific and Technical, 1991.

6. Luongo, A. and Vestroni, F., 'Nonlinear free periodic oscillations of a tethered satellite system', J. Sound and Vibration, 175 (1994) 299-315.

7. Nayfeh, A.H. and Mook, D.T., Nonlinear Oscillations, Wiley-Interscience, New York, 1979.

8. Szemplinska-Stupnicka, W., The Behaviour of Nonlinear Vibration Systems (Volumes I and II), Kluwer Academic Publishers, Dordrecht, The Netherlands, 1990.

9. Ibrahim, R.A. and Barr, A.D.S., 'Autoparametric resonance in a structure cointaining a liquid, part II: three mode interaction', J. Sound and Vibration, 42 (1975) 181-200.

10. Nayfeh, A.H. and Balachadran, B., 'Modal interaction in dynamical and structural systems', Applied Mechanics Reviews, 42 (1989) 175-202.

11. Luongo, A. and Vestroni, F., 'Bifurcations and stability of nonstationary resonant planar oscillations of an orbiting string', Nonlinear Dynamics, 9 (1996) 305-325.

12. Struble, R.A. and Heinbockel, J.H., 'Resonant oscillations of a beam-pendulum system', J. Applied Mechanics, 6 (1963) 181-188.

13. Chakraborty, T. and Rand, R., 'The transition from phase locking to drift in a system of two weakly coupled Van Der Pol oscillators', Int. J. Non-Linear Mechanics, 5/6 (1988) 369-376.

14. Nayfeh, A.H. and Raouf, R.A., 'Nonlinear oscillations of circular cylindrical shells', Int. J. Solids and Structures, 23 (1987) 1625-1638.

15. Lee, C.L. and Perkins, N.C., 'Three-dimensional oscillations of suspended cables involving simultaneous internal resonances'. In: Proceedings of the ASME Winter Annual Meeting, 144, 1992, pp. 59-67.

\section{Appendix A}

The inner product in equations (5) is defined by:

$$
H() \cdot u=\int_{D} H_{D} u \mathrm{~d} D+\int_{\Gamma} H_{\Gamma} u \mathrm{~d} \Gamma
$$


where $H_{D}$ is the formal part of the operator in domain $D$ and $H_{\Gamma}$ is its representation on the boundary $\Gamma$. Accordingly:

$$
\begin{aligned}
& M(\ddot{v}) \cdot \delta v=\int_{0}^{1} \ddot{v} \delta v \mathrm{~d} s+\frac{1}{\gamma} \ddot{v}_{s} \delta v_{s} \\
& L_{v}(v) \cdot \delta v=\int_{0}^{1}\left(f v^{\prime} \delta v^{\prime}-3 \xi x_{0} \delta v\right) \mathrm{d} s-\frac{3}{\gamma} \xi x_{0}(1) \delta v_{s} \\
& L_{w}(v) \cdot \delta w=\int_{0}^{1}\left[f w^{\prime} \delta w^{\prime}+\left(1-3 \xi x_{0}\right) w \delta w\right] \mathrm{d} s+\frac{1}{\gamma}\left(1-3 \xi x_{0}(1)\right) w_{s} \delta w_{s}
\end{aligned}
$$

and

$$
\begin{aligned}
B(v, \dot{v}) \cdot \delta v=- & {\left[\int_{0}^{1} \delta v \int_{0}^{s} v^{\prime} \dot{v}^{\prime} \mathrm{d} s \mathrm{~d} s+\int_{0}^{1} \delta v^{\prime} v^{\prime} \int_{1}^{s} \dot{v} \mathrm{~d} s \mathrm{~d} s\right] } \\
& -\frac{2}{\gamma}\left[\delta v_{s} \int_{0}^{1} v^{\prime} \dot{v}^{\prime} \mathrm{d} s-\dot{v}^{\prime} \int_{0}^{1} \delta v^{\prime} v^{\prime} \mathrm{d} s\right] \\
C(w, \dot{w}) \cdot \delta v= & -2 \int_{0}^{1} \delta v \int_{0}^{s} w^{\prime} \dot{w}^{\prime} \mathrm{d} s \mathrm{~d} s-\frac{2}{\gamma} \delta v_{s} \int_{0}^{1} w^{\prime} \dot{w}^{\prime} \mathrm{d} s \\
D(w, \dot{v}) \cdot \delta w= & -2 \int_{0}^{1} \delta w^{\prime} w^{\prime} \int_{0}^{s} \dot{v} \mathrm{~d} s \mathrm{~d} s+\frac{2}{\gamma} \dot{v}_{s} \int_{0}^{1} \delta w^{\prime} w^{\prime} \mathrm{d} s
\end{aligned}
$$

where $v_{s}=v(1, t), w_{s}=w(1, t)$.

\section{Appendix B}

Table 1. Linear frequencies of transversal modes and spatial frequencies of the modal shapes

\begin{tabular}{lllrrrr}
\hline & & $k=0$ & $k=1$ & $k=2$ & $k=3$ & $k=4$ \\
\hline $1=5 \mathrm{~km}$ & $\omega_{v_{k}}$ & 1.736 & 23.022 & 45.845 & 68.712 & 91.590 \\
& $\omega_{w_{k}}$ & 2.004 & 23.044 & 45.856 & 68.719 & 91.595 \\
& $p_{k}$ & 0.238 & 3.160 & 6.292 & 9.431 & 12.571 \\
$1=100 \mathrm{~km}$ & $\omega_{v_{k}}$ & 1.725 & 6.577 & 12.265 & 18.122 & 24.030 \\
& $\omega_{w_{k}}$ & 1.995 & 6.652 & 12.306 & 18.149 & 24.051 \\
& $p_{k}$ & 0.909 & 3.464 & 6.461 & 9.545 & 12.658 \\
\hline
\end{tabular}

The following values of the system parameters are assumed for the example:

$$
\begin{array}{llrl}
n^{2} & =1.35 \times 10^{-6} \mathrm{~s}^{-2} & & \mu=5.76 \times 10^{-3} \mathrm{~kg} / \mathrm{m} \\
a & =\left(\mu_{e} / n^{2}\right)^{1 / 3}=6657 \mathrm{~km} & A & =4.0 \times 10^{-6} \mathrm{~m}^{2} \\
m & =500 \mathrm{~kg} & E & =7.0 \times 10^{10} \mathrm{~N} / \mathrm{m}^{2} . \\
l & =5 \mathrm{~km} & &
\end{array}
$$


The corresponding nondimensional parameters (1) are:

$$
\alpha^{2}=6.94 \times 10^{-7} ; \quad \gamma=0.0576 ; \quad \xi=7.51 \times 10^{-4}
$$

Frequencies of two systems with different string lengths and the other parameters equal to those of the example are given in Table 1. Spatial frequencies $p_{k}$ of the associated modal shapes of the linearized motion equations $\varphi_{k}(s) \equiv \psi_{k}(s)=\sin p_{k} s$ are also reported.

\section{Appendix C}

The coefficients of equations (12) are as follows:

$$
\begin{array}{ll}
k_{1}=\frac{\omega_{j} b_{k k j}}{4 \omega_{i} m_{i}}, & k_{2}=\frac{\left(\omega_{k}-\omega_{l}\right) c_{k k j}}{4 \omega_{i} m_{i}}, \quad k_{3}=\frac{\omega_{i} b_{k k j}}{4 \omega_{j} m_{j}}, \quad k_{4}=\frac{\omega_{k} c_{j k k}}{4 \omega_{j} m_{j}}, \\
k_{5}=-\frac{\omega_{i} c_{k k j}}{4 \omega_{k} m_{k}}, & k_{6}=\frac{\omega_{j} c_{j k k}}{4 \omega_{k} m_{k}}, \quad k_{7}=-\frac{\omega_{i} c_{k k j}}{4 \omega_{l} m_{l}},
\end{array}
$$

where

$$
\begin{array}{ll}
m_{k}=M\left(h_{k}\right) \cdot h_{k} \quad \text { with } & h=\varphi, \psi \\
b_{i j k}=B\left(\varphi_{j}, \varphi_{k}\right) \cdot \varphi_{i}, \quad & c_{i j k}=C\left(\psi_{j}, \psi_{k}\right) \cdot \varphi_{i}, \quad \mathrm{~d}_{i j k}=D\left(\psi_{j}, \varphi_{k}\right) \cdot \psi_{i} .
\end{array}
$$

\section{Appendix D}

Complex amplitude modulation equations and variational equations for the six-mode model used in Section 7 to analyse the stability of the two-mode non-planar oscillations $\left(a_{k} \neq\right.$ $0, a_{j} \neq 0$ ) are reported:

Governing solution:

$$
\begin{aligned}
& v_{0}(s, t)=A_{i}\left(t_{1}\right) \varphi_{i}(s) e^{i \omega_{k} t_{0}}+A_{j}\left(t_{1}\right) \varphi_{j}(s) e^{i \omega_{j} t_{0}}+A_{r}\left(t_{1}\right) \varphi_{r}(s) e^{i \omega_{r} t_{0}}+\text { c.c. } \\
& w_{0}(s, t)=A_{k}\left(t_{1}\right) \psi_{k}(s) e^{i \omega_{k} t_{0}}+A_{l}\left(t_{1}\right) \psi_{l}(s) e^{i \omega_{l} t_{0}}+A_{s}\left(t_{1}\right) \psi_{s}(s) e^{i \omega_{s} t_{0}}+\text { c.c. }
\end{aligned}
$$

Additional internal resonance conditions:

$$
\begin{aligned}
& \omega_{k}+\omega_{r}-\omega_{s}=\varepsilon \sigma_{4} \\
& \omega_{j}+\omega_{l}-\omega_{s}=\varepsilon \sigma_{5} .
\end{aligned}
$$


$A_{n}$ amplitude equations:

$$
\begin{aligned}
& A_{i}^{\prime}+2 k_{1} \bar{A}_{i} A_{j} e^{-i \sigma_{1} t_{1}}+2 k_{8} \bar{A}_{j} A_{r} e^{-i\left(\sigma_{2}-\sigma_{4}+\sigma_{5}\right) t_{1}}+2 k_{2} \bar{A}_{k} A_{4} e^{-i \sigma_{2} t_{1}}=0 \\
& A_{j}^{\prime}+2 k_{3} A_{i}^{2} e^{i \sigma_{1} t_{1}}+2 k_{9} \bar{A}_{i} A_{r} e^{-i\left(\sigma_{2}-\sigma_{4}+\sigma_{5}\right) t_{1}}+2 k_{4} A_{k}^{2} e^{i \sigma_{3} t_{1}} \\
&+2 k_{10} \bar{A}_{l} A_{g} e^{-i \sigma_{5} t_{1}}=0 \\
& A_{r}^{\prime}+2 k_{11} A_{i} A_{j} e^{i\left(\sigma_{2}-\sigma_{4}+\sigma_{5}\right) t_{1}}+2 k_{12} A_{k} A_{l} e^{i\left(\sigma_{3}-\sigma_{4}+\sigma_{5}\right) t_{1}} \\
&+2 k_{13} \bar{A}_{k} A_{s} e^{-i \sigma_{4} t_{1}}=0 \\
& A_{k}^{\prime}+2 k_{5} \bar{A}_{i} A_{l} e^{-i \sigma_{2} t_{1}}+2 k_{6} \bar{A}_{k} A_{j} e^{-i \sigma_{3} t_{1}}+2 k_{14} \bar{A}_{l} A_{r} e^{-i\left(\sigma_{3}-\sigma_{4}+\sigma_{5}\right) t_{1}} \\
&+2 k_{15} \bar{A}_{\mathrm{r}} A_{s} e^{-i \sigma_{4} t_{1}}=0 \\
& A_{l}^{\prime}+2 k_{7} A_{i} A_{k} e^{i \sigma_{2} t_{1}}+2 k_{16} \bar{A}_{j} A_{s} e^{-i \sigma_{5} t_{1}}+2 k_{17} \bar{A}_{k} A_{r} e^{-i\left(\sigma_{3}-\sigma_{4}+\sigma_{5}\right) t_{1}}=0 \\
& A_{s}^{\prime}+2 k_{18} A_{l} A_{j} e^{i \sigma_{5} t_{1}}+2 k_{19} A_{k} A_{r} e^{i \sigma_{4} t_{1}}=0 .
\end{aligned}
$$

$Z_{n}$ amplitude equations:

$$
\begin{aligned}
& Z_{i}^{\prime}+2 k_{1} \bar{Z}_{i} Z_{j}+2 k_{8} \bar{Z}_{j} Z_{r}+2 k_{2} Z_{l} \bar{Z}_{k}+i \alpha_{i} Z_{i}=0 \\
& Z_{j}^{\prime}+2 k_{3} Z_{i}^{2}+2 k_{9} \bar{Z}_{i} Z_{r}+2 k_{4} Z_{k}^{2}+2 k_{10} \bar{Z}_{k} Z_{l}+i \alpha_{j} Z_{j}=0 \\
& Z_{r}^{\prime}+2 k_{11} Z_{i} Z_{j}+2 k_{12} Z_{k} Z_{l}+2 k_{13} Z_{s} \bar{Z}_{k}+i \alpha_{r} Z_{r}=0 \\
& Z_{k}^{\prime}+2 k_{5} \bar{Z}_{i} Z_{l}+2 k_{6} Z_{j} \bar{Z}_{k}+2 k_{14} Z_{r} \bar{Z}_{l}+2 k_{15} \bar{Z}_{r} Z_{s}+i \alpha_{k} Z_{k}=0 \\
& Z_{l}^{\prime}+2 k_{7} Z_{i} Z_{k}+2 k_{16} \bar{Z}_{j} Z_{s}+2 k_{17} Z_{r} \bar{Z}_{k}+i \alpha_{i} Z_{l}=0 \\
& Z_{s}^{\prime}+2 k_{18} Z_{l} Z_{j}+2 k_{19} Z_{k} Z_{r}+i \alpha_{s} Z_{s}=0 .
\end{aligned}
$$

Additional $\alpha_{n}$ conditions:

$$
\begin{aligned}
& \alpha_{k}+\alpha_{r}-\alpha_{s}+\sigma_{4}=0 \\
& \alpha_{j}+\alpha_{l}-\alpha_{s}+\sigma_{5}=0 .
\end{aligned}
$$

Variational equations:

$$
\begin{aligned}
& \delta Z_{i}^{\prime}+2 k_{1} Z_{j 0} \delta \bar{Z}_{i}+2 k_{8} \bar{Z}_{j 0} \delta Z_{r}+2 k_{2} \bar{Z}_{k_{0}} \delta Z_{l}+i \alpha_{i} \delta Z_{i}=0 \\
& \delta Z_{r}^{\prime}+2 k_{11} Z_{j 0} \delta Z_{i}+2 k_{12} Z_{k_{0}} \delta Z_{l}+2 k_{13} \bar{Z}_{k_{0}} \delta Z_{s}+i \alpha_{r} \delta Z_{r}=0 \\
& \delta Z_{l}^{\prime}+2 k_{7} Z_{k_{0}} \delta Z_{i}+2 k_{16} \bar{Z}_{j 0} \delta Z_{s}+2 k_{17} \bar{Z}_{k_{0}} \delta Z_{r}+i \alpha_{l} \delta Z_{l}=0 \\
& \delta Z_{s}^{\prime}+2 k_{18} Z_{j 0} \delta Z_{l}+2 k_{19} Z_{k_{0}} \delta Z_{r}+i \alpha_{s} \delta Z_{s}=0,
\end{aligned}
$$

where coefficients $k_{i}$ 's are reported in Appendix E. 


\section{Appendix E}

The coefficients of equations (D.3) are as follows:

$$
\begin{aligned}
& k_{1}=\frac{\omega_{j} b_{i i j}}{4 \omega_{i} m_{i}}, \quad k_{8}=\frac{\omega_{r} b_{i j r}-\omega_{j} b_{i r j}}{4 \omega_{i} m_{i}}, \quad k_{2}=\frac{\left(\omega_{l}-\omega_{k}\right) c_{i j i}}{4 \omega_{i} m_{i}}, \quad k_{3}=\frac{\omega_{i} b_{j i i}}{4 \omega_{j} m_{j}} \\
& k_{9}=\frac{\omega_{r} b_{j i r}-\omega_{i} b_{j r i}}{4 \omega_{j} m_{j}}, \quad k_{4}=\frac{\omega_{k} c_{j i i}}{4 \omega_{j} m_{j}}, \quad k_{10}=\frac{\left(\omega_{s}-\omega_{l}\right) c_{j s j}}{4 \omega_{j} m_{j}}, \\
& k_{11}=\frac{\omega_{i} b_{r j i}+\omega_{j} b_{r i j}}{4 \omega_{r} m_{r}} \\
& k_{12}=\frac{\left(\omega_{k}+\omega_{l}\right) c_{r j i}}{4 \omega_{r} m_{r}}, \quad k_{13}=\frac{\left(\omega_{s}-\omega_{k}\right) c_{r i s}}{4 \omega_{r} m_{r}}, \quad k_{5}=-\frac{\omega_{i} d_{i j i}}{4 \omega_{k} m_{k}}, \\
& k_{6}=\frac{\omega_{j} d_{i i j}}{4 \omega_{k} m_{k}} \\
& k_{14}=\frac{\omega_{r} d_{i j r}}{4 \omega_{k} m_{k}}, \quad k_{15}=-\frac{\omega_{r} d_{i s r}}{4 \omega_{k} m_{k}}, \quad k_{7}=\frac{\omega_{i} d_{j i i}}{4 \omega_{l} m_{l}}, \quad k_{16}=-\frac{\omega_{j} d_{j s j}}{4 \omega_{l} m_{l}}, \\
& k_{17}=\frac{\omega_{r} d_{j i r}}{4 \omega_{l} m_{l}} \\
& k_{18}=\frac{\omega_{j} d_{s j j}}{4 \omega_{s} m_{s}}, \quad k_{19}=\frac{\omega_{r} d_{s i r}}{4 \omega_{s} m_{s}} .
\end{aligned}
$$

